\title{
Increased Risk for Carbapenem- Resistant Enterobacteriaceae Colonization in Intensive Care Units after Hospitalization in Emergency Department
}

\author{
Matias Chiarastelli Salomão, Maristela Pinheiro Freire, Icaro Boszczowski,
} Sueli F. Raymundo, Ana Rubia Guedes, Anna S. Levin

Carbapenem-resistant Enterobacteriaceae (CRE) colonization is common in hospital patients admitted to intensive care units (ICU) from the emergency department. We evaluated the effect of previous hospitalization in the emergency department on CRE colonization at ICU admission. Our case-control study included 103 cases and 201 controls; cases were patients colonized by CRE at admission to ICU and controls were patients admitted to ICU and not colonized. Risk factors were emergency department stay, use of carbapenem, Simplified Acute Physiology Score, upper digestive endoscopy, and transfer from another hospital. We found that ED stay before ICU admission was associated with CRE colonization at admission to the ICU. Our findings indicate that addressing infection control problems in EDs will help to control carbapenem resistance in ICUs.

Klebsiella pneumoniae carbapenemase (KPC), deKscribed in 1996, is an enzyme capable of hydrolyzing all $\beta$-lactam antimicrobial drugs known at the time (1). Since then, other carbapenemases have been described in Enterobacteriaceae all over the world, leading to a substantial increase in resistance to antimicrobial drugs $(2,3)$.

Surveillance data from central line-associated bloodstream infections (CLABSI) in intensive care units (ICUs) in the state of São Paulo, Brazil, demonstrated an increase of carbapenem-resistant K. pneumoniae, from 14\% in 2011 to 55\% in 2017 (4). In 2017, K. pneumoniae was the most frequent species causing CLABSI (20\%) in São Paulo.

Author affiliation: Faculdade de Medicina da Universidade de São Paulo, São Paulo, Brazil

DOI: https://doi.org/10.3201/eid2606.190965
Hospital das Clínicas of the University of São Paulo has routinely performed CRE screening for patients admitted to ICU since January 2014. Early identification and isolation of colonized patients was implemented to decrease secondary colonization. Concomitant training sessions for hand hygiene and contact precautions took place during this period. Despite all efforts, ICUs had a high colonization pressure $(17 \%-29 \%$, mean $21 \%)$ due to admission of colonized patients, mainly from EDs (I. Boszczowski, unpub. data).

In 2016, we found that $7 \%$ of patients admitted to the ED were positive for CRE. However, among those who were negative at admission, $18 \%$ became colonized during their stay in the ED. These findings led us to hypothesize that hospitalization in the ED may be a risk factor for CRE colonization in other units of the hospital (5); $\approx 60 \%$ of the patients admitted to ICUs come from hospitalizations in the ED. We evaluated the effect of hospitalization in the ED on CRE colonization at the time of admission to an ICU.

\section{Methods}

\section{Setting}

Hospital das Clinicas is a 2,200-bed public tertiary-care hospital in São Paulo and is the largest hospital complex in Latin America. The main building has $\approx 1,000$ beds and is the location of the ED and most of the hospital's ICU beds (10 ICUs and 109 intensive care beds).

The ED is a very busy unit. In 2018, 69,000 emergency consultations were performed. The average hospitalization rate in the ED is 150 patients/week, and median length of stay is 6 days. The ED has 50 
beds for hospitalization, but occupancy often exceeds 90 beds, with patients on stretchers and often in corridors (Figure 1).

Approximately $60 \%$ of ICU patients are admitted from the ED. To monitor and control CRE colonization, CRE surveillance cultures are performed on all patients admitted to ICUs at the time of admission and placed under contact precautions until the return of results. Colonized patients with CRE remain under contact precautions for their entire stay in the unit.

\section{Microbiology}

Surveillance cultures are performed at the clinical microbiology laboratory in accordance with the institution's standard methodology. Rectal swab specimens from patients are incubated overnight in thioglycolate broth. Positive growth samples are plated on MacConkey agar with ertapenem, imipenem, and meropenem discs. If there are colonies suggestive of Enterobacteriaceae growth within the carbapenems' disk halo, these colonies are isolated and identified by matrix-assisted laser desorption/ ionization time-of-flight mass spectrometry, as recommended by Clinical and Laboratory Standards Institute (6).

\section{Study Design}

We conducted a retrospective case-control study with patients hospitalized in ICUs at HC during September 2015-July 2017. This study used 2 controls for

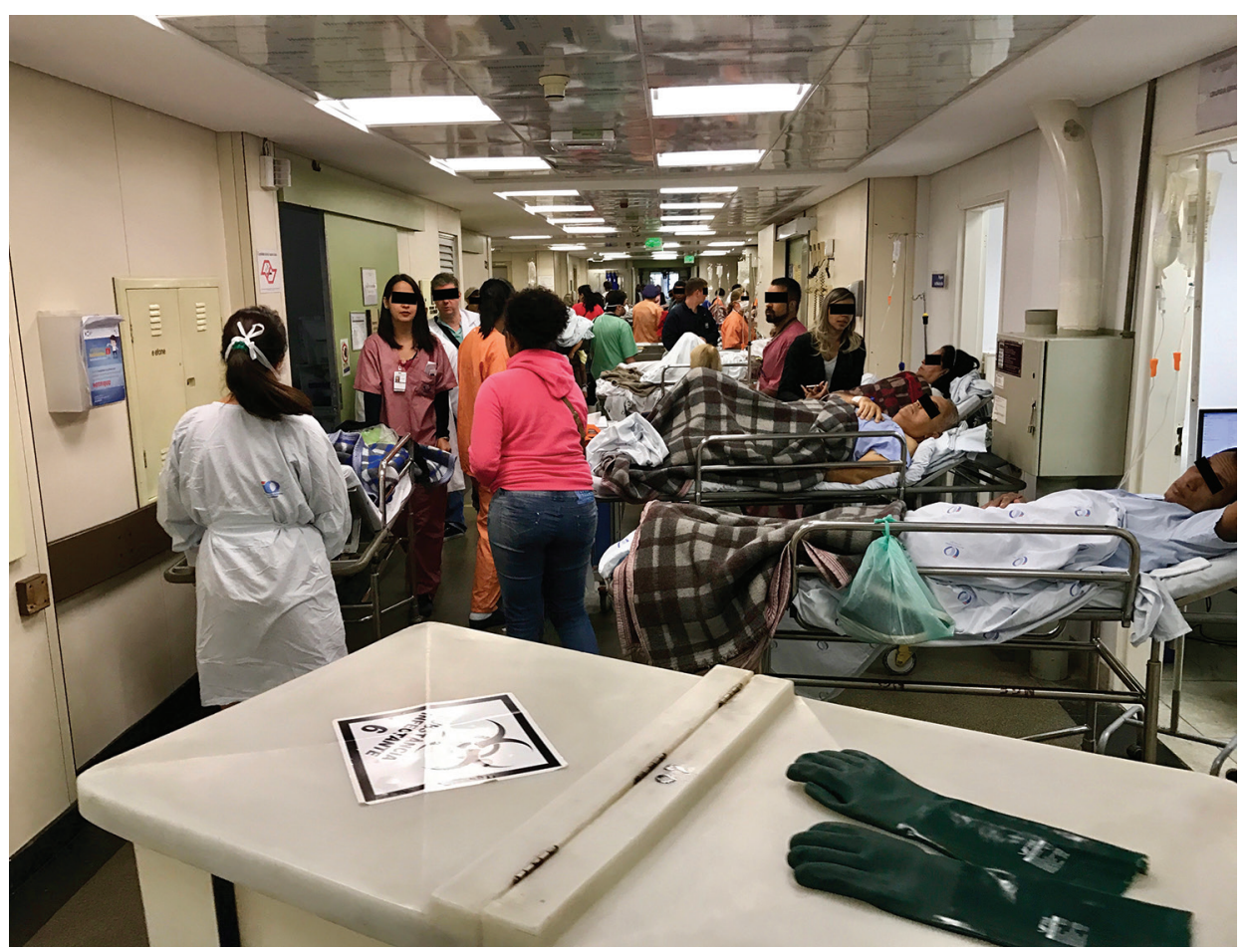

each case. We obtained cases from the infection control department database, which compiles all cases of positive surveillance cultures. Patients who were hospitalized $>1$ time in ICUs were considered only once, during their first hospitalization.

We defined a case as a patient admitted to one of the ICUs during 2015-2017 who had a positive CRE surveillance culture collected within 2 days of admission. We defined a control as a patient admitted to the ICU whose surveillance cultures collected within the first 2 days of admission were negative. Colonization or prior infection with CRE reported at admission were excluding criteria. We paired controls by ICU and hospitalization period, with a maximum interval of 1 week from the admission of the cases. When $>2$ patients were eligible as controls for a case, we randomly chose 2 from all the potential controls. The proportion of controls admitted in the ICUs from the ED was similar to the proportion of patients coming from the ED found in our historical series. CRE screening methodologies were the same for all patients in the study period, whether they were cases or controls.

We collected data from medical records for demographic variables, hospitalization records before ICU admission, clinical characteristics at time of ICU admission, severity scores and organ failures, indwelling devices, clinical procedures before ICU admission, concurrent conditions, use of antimicrobial drugs (for $\geq 48$ hours before ICU admission) and

Figure 1. A corridor in the emergency department of Hospital das Clínicas, São Paulo, Brazil, showing patients on stretchers, December 2016. 
infection before ICU admission, previous colonization, infection by CRE, length of hospital stay, and death. We defined CLABSI according to the 2018 US Centers for Disease Control and Prevention definition (7).

We used REDCap (Research Electronic Data Capture) program (8) to create a data collection tool and database. The Ethics and Research Committee of Hospital das Clinicas da Faculdade de Medicina da Universidade de São Paulo approved this study (number CAAE: 91604518.9.0000.0068).

\section{Statistical Analysis}

We calculated sample size and determined a minimum requirement of 99 cases and 198 controls for $80 \%$ power. We assumed that $35 \%$ of the cases had an ED stay $>2$ days. We performed statistical analysis using Stata version 16 (StataCorp, https://www. stata.com) and SPSS Statistics 11.5 (http:/ / www.ibm. com). We compared cases with controls using the Wilcoxon or McNemar test when appropriate. All tests were 2-tailed, with 95\% CIs; we considered $p<0.05$

Table 1. Characteristics of patients, bivariate analysis, and conditional logistic regression of variables potentially associated with colonization by carbapenem-resistant Enterobacteriaceae at ICU admission, Hospital das Clínicas, São Paulo, Brazil, September 2015-July 2017*

\begin{tabular}{|c|c|c|c|c|c|c|}
\hline \multirow[b]{2}{*}{ Covariate } & \multicolumn{4}{|c|}{ Bivariate analysis } & \multicolumn{2}{|c|}{ Conditional logistic regression } \\
\hline & Cases & Controls & OR $(95 \% \mathrm{Cl})$ & $p$ value & OR $(95 \% \mathrm{Cl})$ & $p$ value \\
\hline Female sex & $34 / 103(33)$ & $91 / 201(45)$ & $0.58(0.35-0.95)$ & 0.03 & & \\
\hline Mean age, y (range) & $50.55(14-84)$ & $49.78(4-89)$ & $1.00(0.99-1.01)$ & 0.62 & & \\
\hline \multicolumn{7}{|l|}{ Previous hospitalization at ICU admission } \\
\hline $\begin{array}{l}\text { Previous stay in another unit } \\
\text { during hospitalization }\end{array}$ & $75 / 101(74)$ & $163 / 201(81)$ & $0.84(0.44-1.60)$ & 0.60 & & \\
\hline $\begin{array}{l}\text { Previous stay in the ED during } \\
\text { hospitalization }\end{array}$ & $62 / 103(60)$ & $125 / 201(62)$ & $1.07(0.65-0.77)$ & 0.78 & & \\
\hline Length of ED stay, d & $2(0-55)$ & $1(0-37)$ & $1.08(1.01-1.15)$ & 0.02 & $1.10(1.02-1.19)$ & 0.01 \\
\hline ED stay $>2 \mathrm{~d}$ & $34 / 103(33)$ & $35 / 201(17)$ & $2.45(1.40-4.32)$ & 0.002 & & \\
\hline Days of hospitalization before & $3(1-95)$ & $2(1-37)$ & $0.99(0.99-0.99)$ & $<0.001$ & & \\
\hline \multicolumn{7}{|l|}{$\begin{array}{l}\text { surveillance culture, median } \\
\text { (range) }\end{array}$} \\
\hline Transfer from another hospital & $43 / 101(43)$ & $51 / 193(26)$ & $2.79(1.26-3.68)$ & 0.005 & $2.52(1.07-5.89)$ & 0.03 \\
\hline Previous hospitalization & $52 / 85(61)$ & $63 / 163(38)$ & $2.91(1.53-5.52)$ & 0.001 & & \\
\hline \multicolumn{7}{|c|}{ Clinical characteristics at ICU admission } \\
\hline Infection & $63 / 101(63)$ & 82/194 (42) & $2.62(1.52-4.54)$ & 0.001 & $1.76(0.56-5.50)$ & 0.33 \\
\hline Sepsis & 46/62 (74) & $54 / 81(66)$ & $1.41(0.52-3.85)$ & 0.50 & & \\
\hline Surgery before ICU admission & $53 / 102(52)$ & $106 / 194(55)$ & 0.92 (0.53-1.62) & 0.78 & & \\
\hline Trauma & $8 / 100(8)$ & 25/194 (13) & $0.62(0.28-1.40)$ & 0.25 & & \\
\hline Stroke & $5 / 100(5)$ & $17 / 194(9)$ & $0.61(0.17-2.18)$ & 0.45 & & \\
\hline \multicolumn{7}{|l|}{ Severity scores } \\
\hline SAPS 3, \% median (range) & $22(4-92)$ & $16(0-98)$ & $1.01(1.002-1.02)$ & 0.01 & $1.01(1.002-1.03)$ & 0.02 \\
\hline SOFA, median (range) & $5(0-19)$ & $5(0-19)$ & $1.09(0.95-1.07)$ & 0.77 & & \\
\hline \multicolumn{7}{|l|}{ Invasive procedures and devices } \\
\hline Dialysis & $14 / 100(14)$ & $11 / 194(6)$ & $2.50(0.97-6.42)$ & 0.06 & & \\
\hline Tracheostomy & 2/99 (2) & $1 / 194(0)$ & $4.92(0.36-44.67)$ & 0.26 & & \\
\hline Colostomy & 2/99 (2) & 2/194 (1) & $2.00(0.28-14.34)$ & 0.49 & & \\
\hline Upper digestive endoscopy & 10/101 (10) & $5 / 194(3)$ & $3.70(1.11-12.32)$ & 0.003 & $18.9(1.83-195.98)$ & 0.01 \\
\hline Colonoscopy & 2/101 (2) & 0/194 (0) & & & & \\
\hline Parenteral nutrition & 2/101 (2) & $1 / 194(1)$ & $3.77(0.19-74.94)$ & 0.38 & & \\
\hline \multicolumn{7}{|l|}{ Underlying conditions } \\
\hline $\mathrm{CCl}$ score, mean (range) & $3.10(0-9)$ & $2.98(0-11)$ & $0.99(0.96-1.02)$ & 0.48 & & \\
\hline Smoking & $25 / 62(40)$ & $46 / 137(34)$ & $1.17(0.49-2.78)$ & 0.72 & & \\
\hline Diabetes mellitus & 20/102 (20) & 44/198 (22) & $0.86(0.46-1.62)$ & 0.65 & & \\
\hline Malignant neoplasm & $9 / 102(9)$ & 23/198 (12) & 0.77 (0.35-1.70) & 0.52 & & \\
\hline $\begin{array}{l}\text { Rheumatologic or autoimmune } \\
\text { disease }\end{array}$ & $11 / 102(11)$ & $16 / 198(8)$ & $1.44(0.66-3.15)$ & 0.36 & & \\
\hline Cirrhosis & $15 / 102(15)$ & $11 / 198(5)$ & $2.25(0.85-5.91)$ & 0.10 & & \\
\hline Chronic kidney disease & $12 / 102(12)$ & 14/198 (7) & $1.51(0.56-3.99)$ & 0.40 & & \\
\hline Solid organ transplant & 8/102 (8) & 16/198 (8) & 0.62 (0.23-1.64) & 0.33 & & \\
\hline HIV infection & $3 / 100(3)$ & $7 / 198(4)$ & $1.13(0.27-4.76)$ & 0.86 & & \\
\hline Hematological malignancy & 2/102 (2) & 6/198 (3) & $0.59(0.13-2.87)$ & 0.52 & & \\
\hline Hematopoietic stem cell transplant & $1 / 102(1)$ & $1 / 198(0)$ & $2.00(0.12-32.42)$ & 0.63 & & \\
\hline \multicolumn{7}{|l|}{ Antimicrobial drug use } \\
\hline Any drug at ICU admission† & 81/99 (81) & 142/193 (71) & $1.56(0.83-2.91)$ & 0.161 & & \\
\hline Carbapenem at ICU admission† & $25 / 80(31)$ & $12 / 141(9)$ & $3.92(1.51-10.21)$ & 0.005 & $4.62(1.30-16.40)$ & 0.02 \\
\hline Any drug use in previous 3 mo & $50 / 72(69)$ & $48 / 145(33)$ & $5.38(2.31-12.53)$ & $<0.001$ & & \\
\hline
\end{tabular}


Carbapenem-Resistant Enterobacteriaceae in ICU

Table 2. Multivariate analysis for potential factors associated with colonization by carbapenem-resistant Enterobacteriaceae at ICU admission, Hospital das Clínicas, São Paulo, Brazil, September 2015-July 2017*

\begin{tabular}{lcc}
\hline Covariate & OR $(95 \% \mathrm{Cl})$ & $\mathrm{p}$ value \\
\hline ED stay $>2 \mathrm{~d}$ & $5.85(1.94-17.65)$ & 0.002 \\
Transfer from another hospital & $2.10(0.95-4.78)$ & 0.076 \\
SAPS 3 score & $1.02(1.003-1.03)$ & 0.02 \\
Carbapenem use on ICU admission, initiated >48 h before ICU admission & $4.78(1.31-17.47)$ & 0.02 \\
Infection at ICU admission & $2.86(1.08-7.55)$ & 0.03 \\
Upper digestive endoscopy & $16.40(2.16-124.50)$ & 0.01 \\
\hline${ }^{*}$ Model using length of ED stay as dichotomous variable. OR, odds ratio; ED, emergency department; ICU, intensive care unit; SAPS 3, Simplified Acute \\
Physiology Score III.
\end{tabular}

statistically significant. For variables with $p<0.05$ in the bivariate analysis, we conducted multivariate analysis with other confounding variables in a conditional logistic regression model. Length of ED stay was a continuous variable and was transformed into a dichotomic variable using SPSS decision tree tool, and for the final model we chose the one with a better fit. We used stepwise backward modeling for the conditional logistic regression and kept the most significant variables in the final model. We used 2 models, one using length of ED stay as a continuous variable and the other as a dichotomous variable. Smoking and sepsis variables comprised more than $40 \%$ of missing data (Tables 1 and 2) and were dropped out.

\section{Results}

We included 304 patients in the study, 103 cases and 201 controls, and collected surveillance cultures for all patients. Of the 103 case-patients, 99 were colonized by K. pneumoniae, 2 by Enterobacter cloacae, and 2 by Escherichia coli. Of the 304 total patients, 188 patients (62\%) were admitted to medical ICUs and 116 (38\%) to surgical ICUs. Sixty-five patients were admitted directly to the ICU: 38 transferred from another hospital, 17 came from the operating room, and for 10 patients, this information was not available. Eightysix patients were transferred from another ward and 152 from the ED; information was not available for 1 patient. Sixty percent of cases and controls stayed in the ED for some time during their hospitalization.

We performed bivariate analysis and demonstrated that 11 characteristics were associated with CRE colonization at ICU admission: sex, ED length of stay, ED stay $>2$ days, number of hospitalization days before the surveillance culture, transfer from another hospital, previous hospitalization, having an infection on ICU admission, clinical severity (SAPS 3 score), use of antimicrobial drugs in the previous 3 months, carbapenem use on ICU admission (initiated $>48$ hours before ICU admission), and upper digestive endoscopy (Table 1). The most common infections at ICU admission were pneumonia (37\%), skin and soft tissue infection (14\%), and CLABSI (10\%).
The median length of stay in the ED was longer for cases ( 2 days, range $0-55)$ than for controls ( 1 day, range $0-37 ; p=0.02$ ) (Figure 2 ). We analyzed the length of stay in the ED with the decision tree tool; we selected a stay $>2$ days as cutoff for this variable $\left(X^{2}=12.799 ; p=0.017\right)$. We found that $38 / 62(61 \%)$ of the patients with CRE colonization at ICU admission were already colonized after 3 days of hospitalization in the ED (Figure 3).

We performed multivariate analysis with 2 models, using ED length of stay as a continuous or a dichotomous variable ( $>2$ days). ED stay was a risk factor for colonization by CRE in both analyses: continuous (per day, odds ratio [OR] 1.10, 95\% CI 1.02$1.19 ; \mathrm{p}=0.01)$ (Table 1 ) and $>2$ days of hospitalization (OR 5.85, 95\% CI 1.94-17.65; p = 0.002) (Table 2). Use of carbapenem at ICU admission (initiated $>48$ hours before ICU admission), Simplified Acute Physiology Score III (SAPS 3), transfer from another hospital, and upper digestive endoscopy were risk factors for CRE colonization at ICU admission (Table 1).

Patients colonized by CRE at ICU admission had higher rates of infection by CRE (18 [18\%]) than did patients not colonized by CRE when they sought care $(11[6 \%] ; p=0.001)$. Colonized patients also had higher in-hospital mortality rates (38 [38\%] for patients colonized by CRE and 48 [24\%] for those not colonized; $\mathrm{p}=0.016$ ).

\section{Discussion}

Our results confirm our hypothesis that ED stay is a risk factor for CRE colonization in patients at the time of admission to the ICU. Other risk factors are use of carbapenem at time of ICU admission (carbapenem use initiated $>48$ hours before ICU admission), SAPS 3 , upper digestive endoscopy, and transfer from another hospital (Table 1).

Including ED stay as a risk factor is a notable new finding. A stay in the ED is usually not considered to be a risk factor for CRE colonization (9). In a previous study, our group demonstrated that patients admitted to the ED had $6.8 \%$ prevalence of CRE colonization at admission to the ED and $18 \%$ acquisition rate for patients hos- 


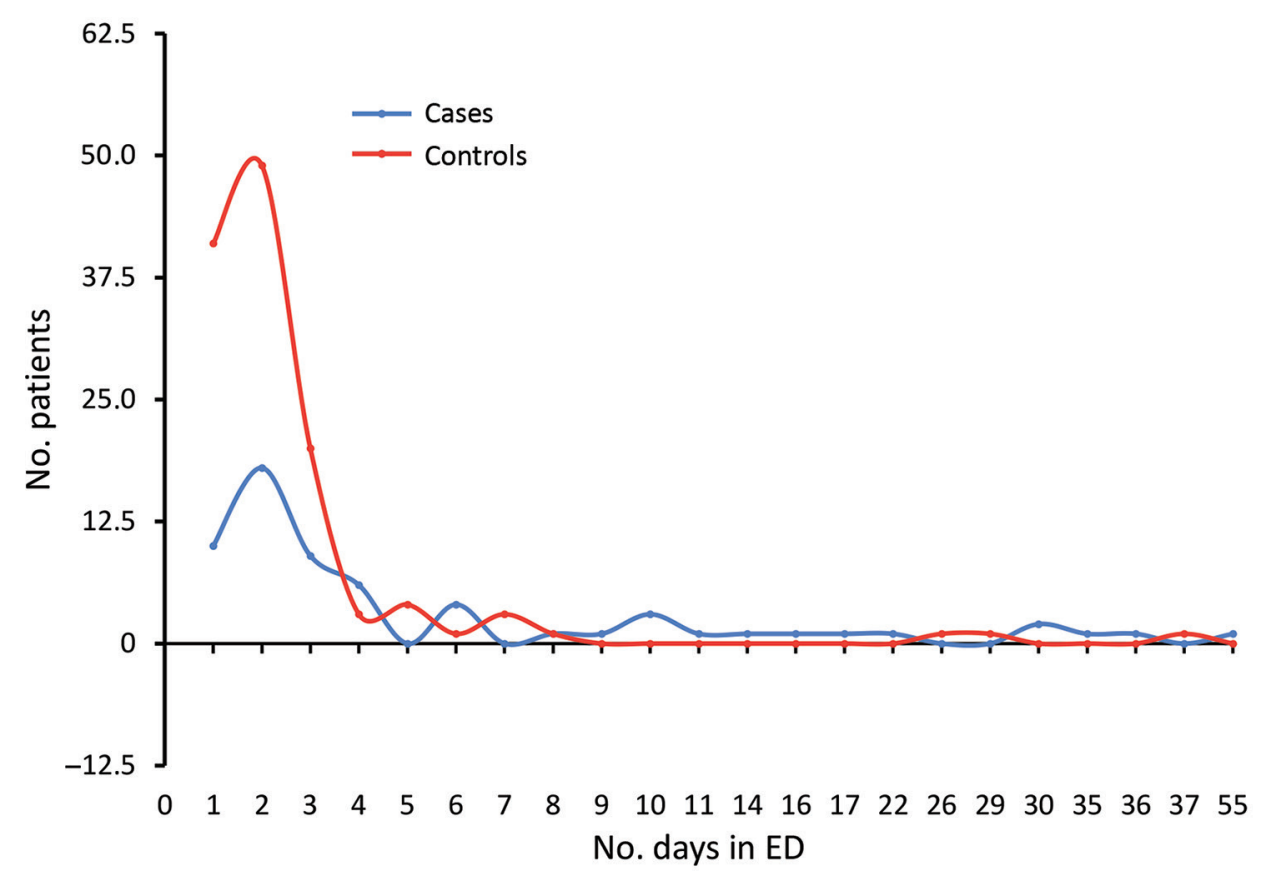

Figure 2. Distribution of days of stay in the emergency department (ED) comparing patients subsequently admitted to an intensive care unit who had a positive carbapenemresistant Enterobacteriaceae culture within 2 days of admission (cases) and patients whose culture was negative (controls), Hospital das Clínicas, São Paulo, Brazil, September 2015-July 2017. pitalized in the ED for longer than 1 week. Six patients who were not treated in a healthcare facility were colonized by CRE at ED admission, implying circulation of this resistance mechanism in the community (5). Our findings show that ED hospitalization is indeed a risk factor for CRE colonization on ICU admission, whereas a previous stay in another hospital unit was not.

Although it is not common, CRE can be found outside the hospital. CRE has been described in community sources of water in Italy (10), Brazil (11), and Sweden (12); in chicken meat in Egypt (13); in vegetables imported from Asia (14), and in hospital sewage in Brazil, China, and Spain (15). Community-acquired CRE infection is difficult to determine; however, up to $30 \%$ of patients with CRE infection on hospital admission have had no previous exposure to the healthcare system.

The acquisition or transmission of CRE in the ED may be a result of the work overload. Ours is a tertiarycare public hospital in Brazil with an overcrowded ED. It is not unusual to have patients with high-complexity illness hospitalized on stretchers for longer than a week because of a shortage of ICU or ward beds to which to transfer patients or to have a low ratio of healthcare workers per patient. Prolonged ED stays probably facilitate cross-transmission of multidrug-resistant organisms such as CRE. Although on first thought the problem may be considered a local one, specific to our hospital and setting, this problem extends to other Brazil hospitals. Two other hospitals reported long stays in the ED, with 1 hospital reporting a median length of stay of 3 days (16) and another reporting that $21 \%$ of patients stayed in the ED for $>5$ days (17). Mortality rates in the EDs of these hospitals are high as well: $7.4 \%$ at the first and $3.9 \%$ at the second. Furthermore, we expect long ED stay is a problem in other countries, although seldom reported (18-20). Lack of access to healthcare in developing countries leads to other problems: healthcare-associated infection rates are much higher in developing countries than in high-income countries (21), as are drug resistance rates (22). In a disadvantaged healthcare system, patients with known risk factors (23-25) are often hospitalized for prolonged periods in the ED and are a potential source of multidrug-resistant bacteria for other patients in the ED and ICUs.

The need to establish strategies to control CRE transmission in EDs and hospitals is urgent; resistance is not an isolated problem in a specific hospital unit or even in a specific hospital. High workload, understaffing, and turnover of healthcare workers make it difficult to improve adherence to hand hygiene in the ED; additional strategies are needed $(26,27)$, and interventions must be multimodal. These interventions must include a change in the workflow of the ED and hospital as well as the entire health system to reduce overcrowding (26-28). The lack of infrastructure in the ED puts patients in stretchers too near to each other, probably facilitating cross-transmission. In this scenario, good hand hygiene may not be achievable. Dividing patients into cohorts and assigning dedicated 


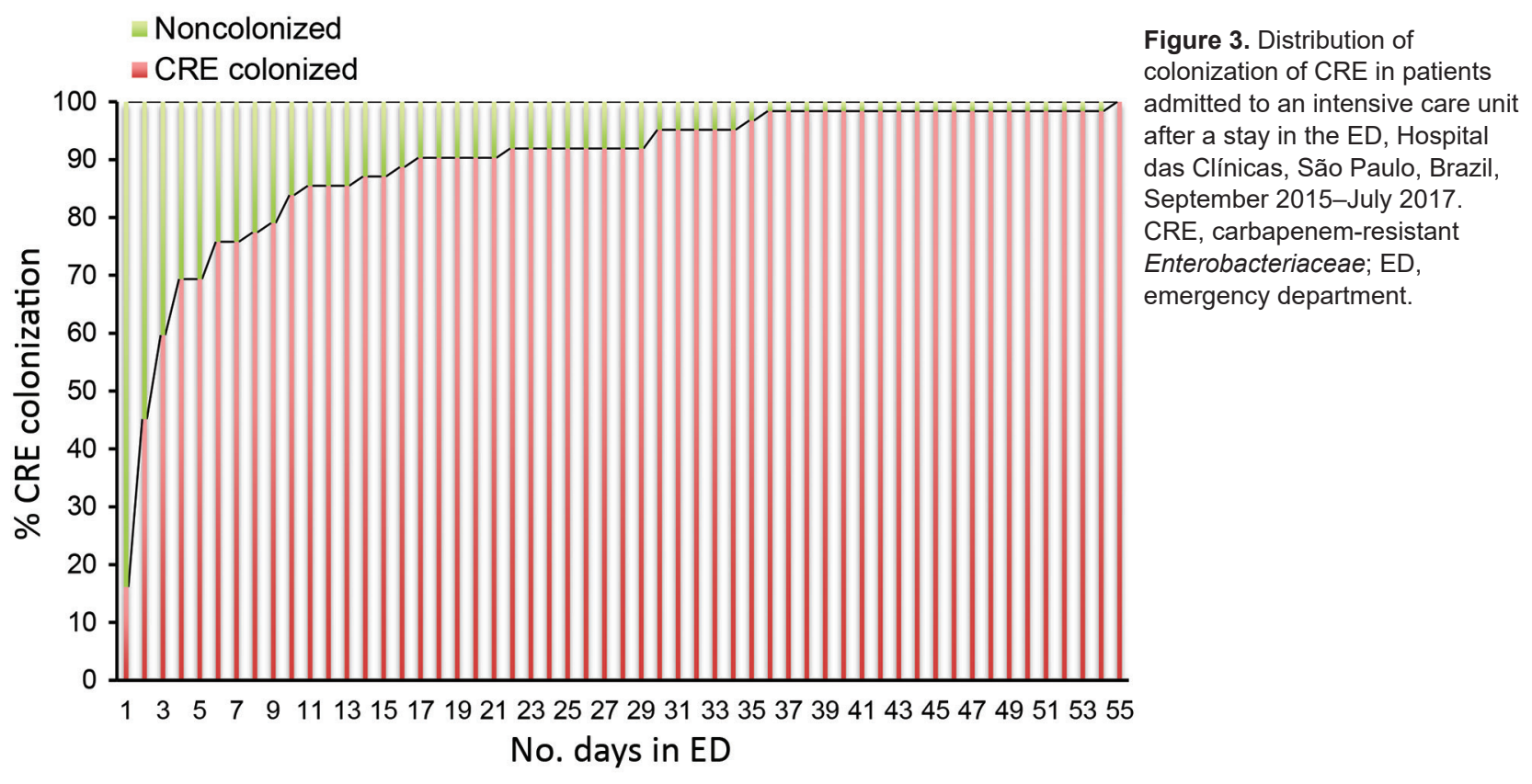

staff may reduce transmission of CRE (29). Hospital staff should discuss screening strategies for CRE and early isolation and contact precautions in the ED (30). Rising antimicrobial resistance is a substantial threat to global health (31), and prolonged ED hospitalization may play a major role in hospital-acquired resistance in low- and middle-income countries.

We found other risk factors that have already been associated with CRE colonization, including transfer from another hospital $(24,25)$, use of carbapenem (23-25), SAPS 3, and upper digestive endoscopy (32). All of them are associated with previous exposure to healthcare or severity of patients (33). The previous use of carbapenems is well described as a risk factor for CRE colonization $(23-25,33)$. In our study, the patients were using carbapenem for $\geq 48$ hours by the time of surveillance culture. Although this timeframe is short, it may have been sufficient for selection of carbapenem-resistant bacteria. We must emphasize that, even though carbapenem use was an independent risk factor in multivariate analysis, the attending physicians may have prescribed it because after a certain length of time in the ED, the patient is at risk for infection by antimicrobial-resistant bacteria.

Of interest, although cirrhosis was not associated with CRE colonization, upper digestive endoscopy was, which suggests that the risk for colonization after endoscopy is probably due to the procedure itself and not to the patient's underlying conditions. We found no clusters of endoscopy-related CRE colonization in the study period, suggesting that it was not an outbreak. Colonization may be a result of improper cleaning procedures. Because this was a retrospective study, we could not test the endoscopes for CRE colonization at the time that colonization occurred. Prospective surveillance for endoscopy-related CRE is underway.

It is difficult to assess the influence of local factors in the hospital ED on colonization by CRE. Factors such as low adherence to hand hygiene and contact precautions, proximity of beds, and others work together to facilitate the transmission of microorganisms. A limitation of this study is that it was not possible to evaluate the effect of each of these variables individually. Other limitations of our study were the retrospective nature of a case-control study; missing data for some variables; potential bias of retrospectively obtaining data from medical records; and the fact that the study was done in only 1 hospital, requiring confirmation in other centers or a multicenter study.

In conclusion, this study demonstrates that prolonged ED stay is a risk factor for CRE colonization at the time of admission to the ICU. Other risk factors were the use of carbapenems at ICU admission (initiated $<48$ hours before ICU admission), SAPS 3 , upper digestive endoscopy, and transfer from another hospital. Clinicians should be aware of the implications of these findings and implement interventions in the ED to control CRE in other hospital units.

\section{Acknowledgments}

We thank the medical archive personnel for providing the medical records, Luizegne Donato for organizing the records and files, and Laina Bubach, Lauro Perdigão, 
and Maria Luisa Moura for helping to review the medical records.

\section{About the Author}

Dr. Salomão is an infectious diseases specialist at the Hospital das Clínicas da Faculdade de Medicina da Universidade de São Paulo. His research focuses on hospital infection control and bacterial resistance.

\section{References}

1. Yigit H, Queenan AM, Anderson GJ, Domenech-Sanchez A, Biddle JW, Steward CD, et al. Novel carbapenemhydrolyzing $\beta$-lactamase, KPC-1, from a carbapenemresistant strain of Klebsiella pneumoniae. Antimicrob Agents Chemother. 2001;45:1151-61. https://doi.org/10.1128/ AAC.45.4.1151-1161.2001

2. Kumarasamy KK, Toleman MA, Walsh TR, Bagaria J, Butt F, Balakrishnan R, et al. Emergence of a new antibiotic resistance mechanism in India, Pakistan, and the UK: a molecular, biological, and epidemiological study. Lancet Infect Dis. 2010;10:597-602. https:/ / doi.org/10.1016/ S1473-3099(10)70143-2

3. Logan LK, Weinstein RA. The epidemiology of carbapenemresistant Enterobacteriaceae: the impact and evolution of a global menace. J Infect Dis. 2017;215(suppl_1):S28-36. https://doi.org/10.1093/infdis/jiw282

4. Center for Epidemiological Surveillance. Data analysis of the epidemiological surveillance system of hospital infections of the state of São Paulo 2017 [in Portuguese]. 2018 [cited 2020 Mar 18]. http:/ / www.saude.sp.gov.br/resources/ cve-centro-de-vigilancia-epidemiologica/areas-devigilancia/infeccao-hospitalar/aulas/ih18_apresentacao_ dados2017.pdf

5. Salomão MC, Guimarães T, Duailibi DF, Perondi MBM, Letaif LSH, Montal AC, et al. Carbapenem-resistant Enterobacteriaceae in patients admitted to the emergency department: new risk factors and occurrence in patients coming directly from the community. J Hosp Infect. 2017;97:241-6._https://doi.org/10.1016/j.jhin.2017.08.012

6. Clinical and Laboratory Standards Institute. Performance standards for antimicrobial susceptibility testing. 26th edition supplement (M100S). Wayne (PA): The Institute; 2006.

7. Centers for Disease Control and Prevention. Bloodstream infection event-central line-associated bloodstream infection and non-central line associated bloodstream infection. Device-associated module. 2020 [cited 2020 Mar 27]. /https://www.cdc.gov/nhsn/pdfs/pscmanual/ 4psc_clabscurrent.pdf

8. Harris PA, Taylor R, Thielke R, Payne J, Gonzalez N, Conde JG. Research electronic data capture (REDCap) - a metadata-driven methodology and workflow process for providing translational research informatics support. J Biomed Inform. 2009;42:377-81. https:/ / doi.org/10.1016/ j.jbi.2008.08.010

9. Richter SS, Marchaim D. Screening for carbapenemresistant Enterobacteriaceae: who, when, and how? Virulence. 2017;8:417-26. https:/ / doi.org/10.1080/ 21505594.2016.1255381

10. Caltagirone M, Nucleo E, Spalla M, Zara F, Novazzi F, Marchetti VM, et al. Occurrence of extended spectrum $\beta$-lactamases, KPC-type, and MCR-1.2-producing Enterobacteriaceae from wells, river water, and wastewater treatment plants in Oltrepò Pavese area, Northern Italy. Front Microbiol. 2017;8:2232. https://doi.org/10.3389/ fmicb.2017.02232

11. Bartley PS, Domitrovic TN, Moretto VT, Santos CS, Ponce-Terashima R, Reis MG, et al. Antibiotic resistance in Enterobacteriaceae from surface waters in urban Brazil highlights the risks of poor sanitation. Am J Trop Med Hyg. 2019;100:1369-77.

12. Khan FA, Hellmark B, Ehricht R, Söderquist B, Jass J. Related carbapenemase-producing Klebsiella isolates detected in both a hospital and associated aquatic environment in Sweden. Eur J Clin Microbiol Infect Dis. 2018;37:2241-51. https://doi.org/10.1007/s10096-018-3365-9

13. Abdallah HM, Alnaiemi N, Reuland EA, Wintermans BB, Koek A, Abdelwahab AM, et al. Fecal carriage of extendedspectrum $\beta$-lactamase- and carbapenemase-producing Enterobacteriaceae in Egyptian patients with community-onset gastrointestinal complaints: a hospital-based cross-sectional study. Antimicrob Resist Infect Control. 2017;6:62. https://doi.org/10.1186/s13756-017-0219-7

14. Zurfluh K, Poirel L, Nordmann P, Klumpp J, Stephan R. First detection of Klebsiella variicola producing OXA-181 carbapenemase in fresh vegetable imported from Asia to Switzerland. Antimicrob Resist Infect Control. 2015;4:38. https:/ / doi.org/10.1186/s13756-015-0080-5

15. Kelly AM, Mathema B, Larson EL. Carbapenem-resistant Enterobacteriaceae in the community: a scoping review. Int J Antimicrob Agents. 2017;50:127-34. https://doi.org/ 10.1016/j.ijantimicag.2017.03.012

16. Peres RR, Lima SBS, de Souza Magnago TSB, Shardong AC, da Silva Ceron MD, Prochnow A, et al. Clinicalepidemiological profile of patients admitted to the emergency department of a university hospital [in Portuguese]. Rev Saúde (Santa Maria). 2013;39:77-86.

17. Ribeiro RCHM, Rodrigues CC, Canova JCM, Rodrigues CDS, Cesarino CB, Júnior OLS. Stay and outcome of the clinical and surgical patient in the emergency service. Rev Enferm (Lisboa). 2013;7:5426-32. https:// periodicos.ufpe.br/revistas/ revistaenfermagem/article/download/13670/16557

18. Phua J, Ngerng WJ, Lim TK. The impact of a delay in intensive care unit admission for community-acquired pneumonia. Eur Respir J. 2010;36:826-33. https://doi.org/ 10.1183/09031936.00154209

19. Cardoso LT, Grion CM, Matsuo T, Anami EH, Kauss IA, Seko L, et al. Impact of delayed admission to intensive care units on mortality of critically ill patients: a cohort study. Crit Care. 2011;15:R28. https:/ / doi.org/10.1186/cc9975

20. Obermeyer Z, Abujaber S, Makar M, Stoll S, Kayden SR, Wallis LA, et al.; Acute Care Development Consortium. Emergency care in 59 low- and middle-income countries: a systematic review. Bull World Health Organ. 2015;93:577586G. https://doi.org/10.2471/BLT.14.148338

21. Rosenthal VD, Maki DG, Mehta Y, Leblebicioglu H, Memish ZA, Al-Mousa HH, et al.; International Nosocomial Infection Control Consortium. International Nosocomial Infection Control Consortium (INICC) report, data summary of 43 countries for 2007-2012. Device-associated module. Am J Infect Control. 2014;42:942-56. https://doi.org/10.1016/ j.ajic.2014.05.029

22. World Health Organization. Antimicrobial resistance global report on surveillance. Geneva: The Organization; 2014 [cited 2020 Mar 18]. http:/ / apps.who.int/iris/ bitstream/10665/112642/1/9789241564 748_eng.pdf

23. Papadimitriou-Olivgeris M, Marangos M, Fligou F, Christofidou M, Bartzavali C, Anastassiou ED, et al. Risk factors for KPC-producing Klebsiella pneumoniae enteric 
colonization upon ICU admission. J Antimicrob Chemother. 2012;67:2976-81.

24. Marchaim D, Chopra T, Bhargava A, Bogan C, Dhar S, Hayakawa K, et al. Recent exposure to antimicrobials and carbapenem-resistant Enterobacteriaceae: the role of antimicrobial stewardship. Infect Control Hosp Epidemiol. 2012;33:817-30. https:/ / doi.org/10.1086/666642

25. Schwaber MJ, Klarfeld-Lidji S, Navon-Venezia S,

Schwartz D, Leavitt A, Carmeli Y. Predictors of carbapenemresistant Klebsiella pneumoniae acquisition among hospitalized adults and effect of acquisition on mortality. Antimicrob Agents Chemother. 2008;52:1028-33. https:// doi.org/10.1128/AAC.01020-07

26. Carter EJ, Wyer P, Giglio J, Jia H, Nelson G, Kauari VE, et al. Environmental factors and their association with emergency department hand hygiene compliance: an observational study. BMJ Qual Saf. 2016;25:372-8. https:/ / doi.org/ 10.1136/bmjqs-2015-004081

27. Muller MP, Carter E, Siddiqui N, Larson E. Hand hygiene compliance in an emergency department: the effect of crowding. Acad Emerg Med. 2015;22:1218-21. https://doi.org/10.1111/acem.12754

28. Yarmohammadian MH, Rezaei F, Haghshenas A, Tavakoli N. Overcrowding in emergency departments: a review of strategies to decrease future challenges. J Res Med Sci. 2017;22:23.

29. Legeay C, Thépot-Seegers V, Pailhoriès H, Hilliquin D, Zahar JR. Is cohorting the only solution to control carbapenemase-producing Enterobacteriaceae outbreaks? A single-centre experience. J Hosp Infect. 2018;99:390-5. https:/ / doi.org/10.1016/j.jhin.2018.02.003

30. Munoz-Price LS, Quinn JP. Deconstructing the infection control bundles for the containment of carbapenem-resistant Enterobacteriaceae. Curr Opin Infect Dis. 2013;26:378-87. https://doi.org/10.1097/01.qco.0000431853.71500.77

31. Centers for Disease Control and Prevention. Antibiotic resistance threats in the United States, 2013 [cited 2020 Mar 27]. https:/ / www.cdc.gov/drugresistance/pdf/ ar-threats-2013-508.pdf

32. Muscarella LF. Risk of transmission of carbapenem-resistant Enterobacteriaceae and related "superbugs" during gastrointestinal endoscopy. World J Gastrointest Endosc. 2014;6:457-74. https:// doi.org/10.4253/wjge.v6.i10.457

33. van Loon K, Voor In't Holt AF, Vos MC. A systematic review and meta-analyses of the clinical epidemiology of carbapenem-resistant Enterobacteriaceae. Antimicrob Agents Chemother. 2017;62:e01730-17. https://doi.org/10.1128/ AAC.01730-17

Address for correspondence: Matias Chiarastelli Salomão,

Av Dr Eneas de Carvalho Aguiar, 255, Instituto Central Hospital das Clínicas da Faculdade de Medicina da Universidade de São Paulo, 5o andar, Subcomissão de Controle de Infecção Hospitalar, São Paulo/SP, CEP 05403-000, Brazil; email: matias.salomao@hc.fm.usp.br

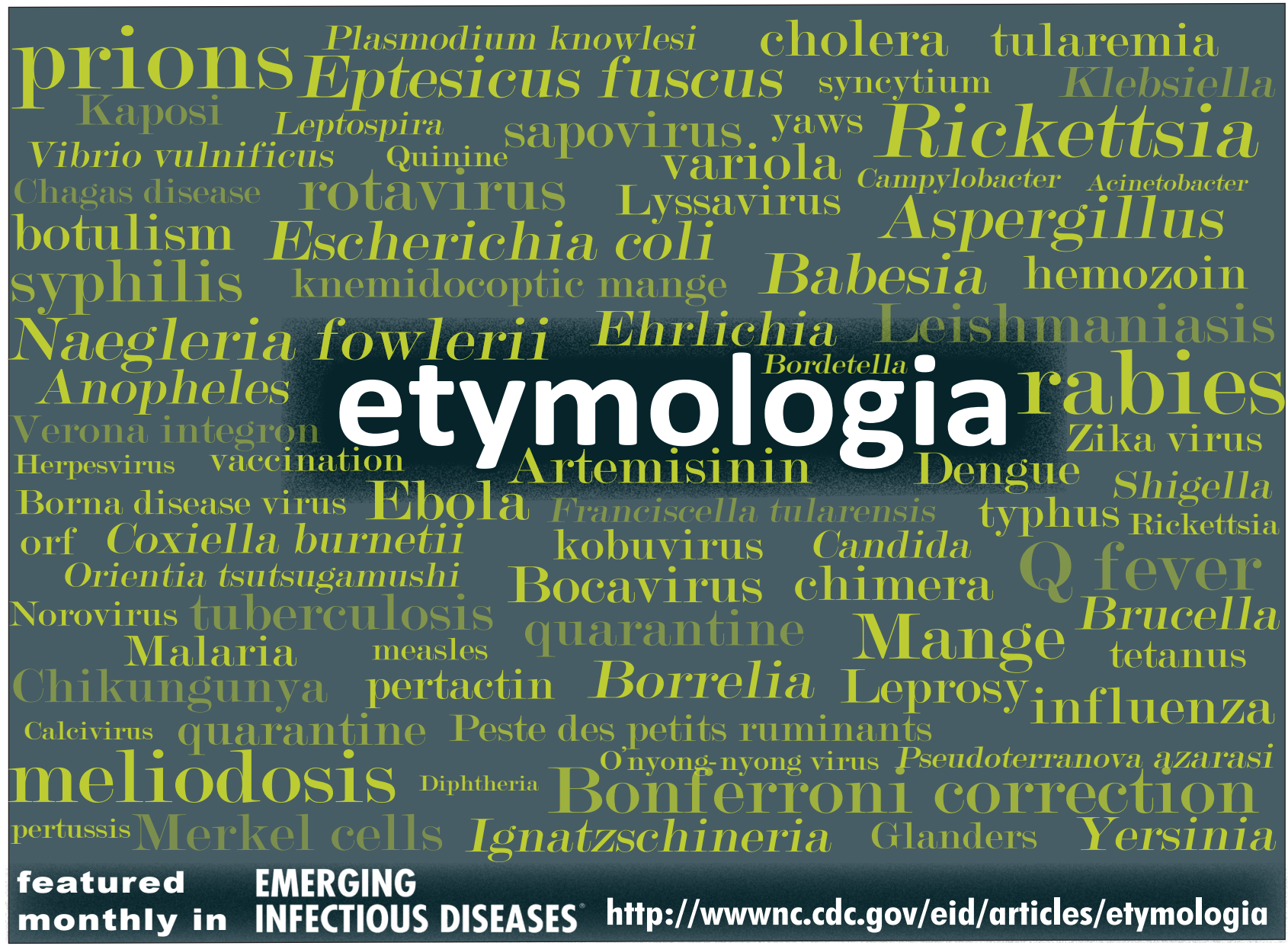

